\title{
L'évolution du système éducatif rwandais à travers un siècle de réformes
}

\section{Masengesho Kamuzinzi}

\section{OpenEdition}

1 Journals

Édition électronique

URL : http://journals.openedition.org/ries/2251

DOI : 10.4000/ries.2251

ISSN : 2261-4265

Éditeur

Centre international d'études pédagogiques

Édition imprimée

Date de publication : 1 avril 2012

Pagination : 25-31

ISBN : 978-2-85420-594-7

ISSN : $1254-4590$

Référence électronique

Masengesho Kamuzinzi, "L'évolution du système éducatif rwandais à travers un siècle de réformes ", Revue internationale d'éducation de Sèvres [En ligne], 59 | avril 2012, mis en ligne le 01 avril 2015,

consulté le 01 mai 2019. URL : http://journals.openedition.org/ries/2251 ; DOI : 10.4000/ries.2251

Ce document a été généré automatiquement le 1 mai 2019.

(c) Tous droits réservés 


\title{
L'évolution du système éducatif rwandais à travers un siècle de réformes
}

\author{
Masengesho Kamuzinzi
}

1 Cet article retrace les différents traits des réformes ayant marqué le système éducatif rwandais, depuis sa naissance en 1900 jusqu'à nos jours. Pendant huit décennies, le réseau public et libre subventionné a fonctionné sur la base d'une philosophie pseudo-élitiste consistant à sélectionner des candidats issus surtout du groupe ethnique politiquement privilégié selon l'époque, auxquels l'état garantissait par la suite des places dans son administration, et à verser les déclassés dans des filières rurales et artisanales sans perspective. À partir de la décennie quatre-vingt, la montée d'aspirations citoyennes a contraint le pouvoir en place à consentir à plus d'ouverture, faisant naitre une troisième force à côté de l'État et des Églises : les associations de parents. Pendant les décennies qui ont suivi cette prise de conscience, le système éducatif rwandais est passé d'un extrême à l'autre, c'est-à-dire de l'obsession de contrôle de l'accès à une "anarchie d'initiatives ", d'abord au niveau du secondaire depuis 1982, puis au niveau du supérieur après le génocide ${ }^{1}$ de 1994. Le foisonnement d'initiatives, après cette période cruciale de l'histoire nationale, a marqué une rupture avec la philosophie éducative du passé et a poussé, ces dernières années, l'État rwandais à renforcer ses mécanismes de régulation.

\section{0-1959 : élitisme colonial vs école de « masse » rurale}

2 Le système éducatif rwandais inauguré par les colons allemands prit la forme de quelques « chapelles-écoles » dédiées essentiellement au catéchisme. Le passage sous tutelle belge, après la Première Guerre mondiale, fut un tournant décisif qui consacra le monopole de l'Église catholique sur l'organisation des enseignements. La commission réunie autour du ministre des colonies Frank, en 1922, confia le soin d'organiser l'enseignement aux 
«missions» essentiellement catholiques. Cette convention fut étendue aux autres missions chrétiennes, à partir de l'intervention du ministre libéral Godding en 1946. Sur le plan légal, ce nouveau dispositif consistait à passer des conventions à long terme avec les Églises, en leur accordant de larges subventions contre un respect de la réglementation et des programmes définis par l'État belge (Erny, 1974).

3 Le système éducatif mis en place alors se caractérise par une sélectivité précoce et une absence de passerelles. Il offre deux perspectives aux avantages incomparables: une formation destinée à l'élite "subalterne ", à laquelle sont garanties des places dans l'administration coloniale, et une formation destinée à la "masse ", à caractère rural et sans débouché. Tous les dispositifs réglementaires qui se succèdent de 1925 à 1948 confirment cette dichotomie. La réforme de 1948 va jusqu'à proposer une sorte de "spécialisation ", après la deuxième année du primaire, en instituant deux filières : l'une dite " ordinaire ", consacrée à la masse, et l'autre dite « sélectionnée », consacrée à l'élite.

4 Au plan sociopolitique, l'école dite d'élite visait en premier lieu à faire basculer en douceur l'élite traditionnelle au service de la colonisation, en perpétuant ses avantages, comme le montre le choix de la tutelle belge de consacrer certaines écoles à la formation des fils des chefs et des sous-chefs sélectionnés, afin qu'ils puissent seconder leurs pères, une fois leur formation terminée. Quant à l'école de masse, qui ne recueillait par ailleurs que quelques milliers d'enfants avant l'indépendance, le primat de la ruralisation de l'enseignement était vécu comme une sorte de maintien du statu quo.

\section{De l'indépendance à 1980 : quelle école de masse?}

5 Bien que l'accession du Rwanda à l'indépendance, le 1er juillet 1962, ait favorisé l'accès au pouvoir d'un parti républicain, le MDR-Paremehutu, dont le leitmotiv, en matière d'éducation, était l'abolition des privilèges dont avait bénéficié l'élite monarchiste, dans les faits, le système éducatif post-indépendance ne renonce pas fondamentalement à la dichotomie élitisme vs ruralisation. En effet, le congrès du MDR-Parmehutu de 1969 reprend à son compte le discours de 1948 et recommande une révision des programmes afin que les nombreux élèves qui ne peuvent accéder au secondaire puissent terminer l'école primaire après avoir été habitués à travailler la terre (Erny, 1974).

Bien que cette réforme se soit heurtée à une forte résistance, on assiste à un retour des telles préoccupations dans la première réforme proposée par les autorités de la deuxième République en 1973. Cette réforme revient sur le fait que les programmes du primaire sont essentiellement orientés vers le passage dans l'enseignement secondaire, alors que plus de $90 \%$ des enfants qui commencent le cycle primaire doivent retourner vivre sur la colline. La nouvelle réforme se propose non seulement d'adapter les programmes à la vie rurale mais également de retarder l'âge d'admission jusqu'à 9 ans afin qu'en achevant leur cycle primaire, vers 16 ans, ces jeunes soient en mesure de se lancer directement dans un travail agricole productif!

Elle revient également sur une proposition de 1948 séparant l'école de l'« élite » de l'école de la "masse", en proposant un cycle inférieur de quatre ans consacré à l'alphabétisation, au terme duquel interviendrait un concours permettant de retenir une soixantaine d'enfants par commune pour effectuer un deuxième cycle donnant accès au 
secondaire, les autres élèves étant versés dans un deuxième cycle de masse consacré à la formation familiale, agricole et artisanale.

La réforme de 1979 n'est pas exempte de telles visées. Elle revoit l'âge d'admission à 7 ans mais prolonge la durée du cycle primaire à huit années, les deux dernières étant consacrées à la formation agricole et artisanale. Désormais, la sélection des élèves devant entrer à l'école d'« élite » et de «masse » s'opère à la fin du cycle primaire en combinant trois critères : les résultats au concours, le quota ethnique et le quota régional. Mais ce lourd dispositif ne permet en fait qu'à $10 \%$ des lauréats du primaire d'accéder au secondaire (Rugengande, 2008).

\section{Désenchantement de la ruralisation comme voie d'accès de la masse à l'éducation}

9 Dans un système sans diversification ni passerelle, l'application des trois critères cités précédemment offrait aux élèves deux destins clairement opposés: l'espoir d'une promotion sociale et un accès futur à l'emploi d'une part, et l'absence de perspective d'avenir, d'autre part. Pour les familles les plus tenaces, la lutte pour faire accéder leurs enfants au secondaire exigeait des stratégies officieuses, dont le changement d'école et de date de naissance, le changement d'ethnie dans l'espoir de contourner le quota ethnique censé attribuer de facto $90 \%$ des places aux enfants hutus, $9 \%$ aux enfants tutsis et $1 \%$ aux enfants twas étant la stratégie la plus osée dans cette course pour décrocher une place dans l'école de l'élite.

Bien que la ruralisation de l'enseignement de masse fût présentée, dans le discours officiel, comme le meilleur moyen de réconcilier l'enfant avec son milieu, l'acharnement à limiter l'accès à l'école d'élite renforçait la conviction des déclassés que cette option était une manière de les maintenir dans un statut inférieur, les excluant de fait de la direction future du pays.

\section{L'émergence d'une troisième voie : l'initiative privée}

11 La décennie 1980 marque un début de rupture avec cette logique pseudo-élitiste. Des pressions internes, alimentées par la frustration des parents des déclassés, associées aux pressions externes appelant l'État à alléger le poids des dépenses d'éducation sur le budget, conduisent à libéraliser progressivement l'organisation de l'enseignement.

En 1981/1982, les premières écoles privées sont ouvertes par des associations de parents avant même d'y être invitées par l'État. Sept ans plus tard, leur nombre dépasse celui des écoles du réseau public et libre vieux de neuf décennies. Conscientes de constituer une voie alternative de promotion sociale non contrôlée par le pouvoir politique, ces écoles se focalisent surtout sur les filières professionnelles très prisées à l'époque, comme les sciences infirmières, le commerce et la comptabilité, le droit et l'administration, les sciences économiques, etc. 


\section{Changements intervenus après le génocide}

13 Les changements intervenus après le génocide de 1994 se remarquent à trois niveaux différents : l'explosion de l'accès, la diversification des programmes d'enseignement et l'introduction de la spécialisation dans la formation des enseignants.

14 En ce qui concerne l'accroissement de l'accès, la combinaison de quatre facteurs clés met fin à la promotion « sur mesure » qui caractérisait le système depuis sa naissance.

Le premier facteur a trait à l'alignement de la politique éducative sur les directives internationales d'éducation pour tous, comme en témoigne l'encadré 1 .

16 Avec l'adoption de la politique dite «nine years basic education » en 2008, le système éducatif rwandais a, pour la première fois depuis sa création, commencé à offrir un enseignement secondaire gratuit, la politique éducative actuelle étant d'étendre ce réseau d'enseignement gratuit jusqu'à la fin du cycle secondaire. Ce choix a donné un coup d'accélérateur à la construction des nouvelles écoles et à l'accroissement des effectifs qui ont doublé en l'espace de cinq ans (encadré 2).

Le deuxième facteur a trait au choix du gouvernement de bannir la politique des quotas et de décréter la réussite au concours comme le seul critère d'accès à l'enseignement secondaire, supérieur et universitaire.

Encadré $\mathrm{n}^{\circ} 1$ : évolution des indicateurs d'accès au niveau primaire entre 2005-2010

\begin{tabular}{|l|c|c|c|c|c|c|}
\hline Année & $\mathbf{2 0 0 5}$ & $\mathbf{2 0 0 6}$ & $\mathbf{2 0 0 7}$ & $\mathbf{2 0 0 8}$ & $\mathbf{2 0 0 9}$ & $\mathbf{2 0 1 0}$ \\
\hline Effectifs d'élèves & 1857841 & 2019991 & 2150430 & 2190270 & 2264672 & 2299326 \\
\hline \% garçons & $49,1 \%$ & $48,7 \%$ & $49,2 \%$ & $49,1 \%$ & $49,2 \%$ & $49,3 \%$ \\
\hline \% filles & $50,9 \%$ & $51,3 \%$ & $50,8 \%$ & $50,9 \%$ & $50,8 \%$ & $50,7 \%$ \\
\hline Taux brut de scolarisation & $137,3 \%$ & $145,3 \%$ & $151,9 \%$ & $127,9 \%$ & $128,5 \%$ & $126,5 \%$ \\
\hline Taux net de scolarisation & $93,5 \%$ & $95,0 \%$ & $95,8 \%$ & $94,2 \%$ & $92,9 \%$ & $95,4 \%$ \\
\hline Nombre d'enseignants & 26,944 & 28,474 & 29,059 & 33,158 & 32,412 & 33,842 \\
\hline Ratio élèves/maître & 74 & 73 & 75 & 73 & 63 & 63 \\
\hline
\end{tabular}

Source : Mineduc (2010)

Encadré $n^{\circ} 2$ : évolution des indicateurs d'accès au niveau secondaire entre 2005-2010

\begin{tabular}{|l|c|c|c|c|c|c|}
\hline Année scolaire & $\mathbf{2 0 0 5}$ & $\mathbf{2 0 0 6}$ & $\mathbf{2 0 0 7}$ & $\mathbf{2 0 0 8}$ & $\mathbf{2 0 0 9}$ & $\mathbf{2 0 1 0}$ \\
\hline Effectifs d'élèves & 218517 & 239629 & 266518 & 288036 & 346518 & 425587 \\
\hline$\%$ garçons & $52,8 \%$ & $52,5 \%$ & $52,4 \%$ & $52,2 \%$ & $51,0 \%$ & $49,3 \%$ \\
\hline$\%$ filles & $47,2 \%$ & $47,5 \%$ & $47,6 \%$ & $47,8 \%$ & $49,0 \%$ & $50,7 \%$ \\
\hline Nombre d'enseignants & 7,610 & 7,818 & 12,103 & 10,187 & 14,426 & 14,477 \\
\hline Nombre d'écoles & 553 & 579 & 643 & 689 & 686 & 1,399 \\
\hline
\end{tabular}

Source : Mineduc (2010)

18 Le troisième facteur est l'explosion des l'initiatives privées, surtout au niveau supérieur dont les effectifs passent de quelques centaines d'étudiants répartis dans quatre établissements en 1994 à 31170 étudiants encadrés par treize universités et instituts 
supérieurs en 2010. Le réseau d'enseignement supérieur public se développe également de manière spectaculaire après le génocide. En 2010, le Rwanda compte dix-sept institutions d'enseignement supérieur publiques pour 31564 étudiants.

La ruée vers les universités et les instituts supérieurs publics et privés après le génocide finit par diminuer l'attrait du diplôme du secondaire, occasionnant une nouvelle fièvre en faveur du diplôme de licence, qui est devenu progressivement le niveau de référence de recrutement dans le secteur public et privé. Le marché de l'emploi devenant saturé, la recherche d'un diplôme de master s'observe particulièrement chez les cadres soucieux de se maintenir à leurs postes. Ainsi, depuis 2005, les universités des pays limitrophes organisant des programmes de master durant le week-end sont littéralement prises d'assaut par des fonctionnaires rwandais, obligeant les universités publiques et privées du Rwanda à se lancer dans cette nouvelle aventure d'organisation des programmes de masters. À cela s'ajoute la création, ces dernières années, de «succursales » des universités internationales déjà présentes dans les pays de l'Afrique de l'Est, comme la Mont Kenya University, Carnegie Mellon University in Rwanda, etc.

Le quatrième facteur a trait à l'allégement du dispositif réglementaire. À la faveur du climat de reconstruction post-génocide, la plupart des écoles et universités privées fonctionnaient d'abord sans accréditation. Elles n'en sollicitaient une que lorsqu'elles étaient sûres d'avoir réuni les conditions des exigences légales.

21 En ce qui concerne les changements intervenus dans la conception des programmes, le Rwanda post-génocide présente la particularité d'avoir hébergé plus ou moins tacitement plusieurs systèmes « exportés » par les anciens exilés venant des pays limitrophes et dont les enfants ne parvenaient à s'insérer dans le système existant. Si l'harmonisation au niveau primaire présentait moins de défis, en raison des similarités observées dans les programmes en vigueur au Rwanda et dans les pays limitrophes, il n'en était pas de même du niveau secondaire et supérieur. Ainsi, certaines écoles de la province de l'Est ayant recueilli les élèves revenus de l'Ouganda et de la Tanzanie ont continué, pendant plusieurs années, à suivre les programmes des pays de provenance, alors que ceux venus du Burundi et de la République démocratique du Congo se sont tant bien que mal insérés dans le système existant.

Pendant plus d'une décennie, le système éducatif rwandais balance entre deux traditions souvent qualifiées de « francophone » et d'« anglophone ». Le système " francophone » en vigueur au Rwanda avant le génocide était notamment caractérisé par la diversification des matières au secondaire. Presque toutes les sections avaient trois cours d'option, trois cours de culture générale - dont l'histoire, la géographie et l'éducation civique -, et trois cours des sciences. À cela s'ajoutaient des cours d'ordre général, comme la religion et l'éducation physique, et parfois l'économie domestique et l'écologie. La seconde tradition, inspirée des pays anglophones de l'Afrique de l'Est, était caractérisée notamment par la réduction du nombre des matières au niveau primaire et par la focalisation sur trois matières spécifiques, en fonction des sections disponibles au secondaire.

À partir de 2007, le Rwanda adopte finalement cette seconde perspective, à la faveur de son entrée dans la Communauté des États de l'Afrique de l'Est. Quatre matières principales sont enseignées au premier cycle du primaire : l'anglais, le Kinyarwanda, les mathématiques élémentaires et les social studies. Les autres matières, dont la religion, la musique, le modelage, les activités culturelles et les travaux manuels, se partagent seulement quatre heures par semaine. Au niveau du deuxième cycle s'ajoute le cours de 
technologies élémentaires. Hormis les cours des langues, les matières sont enseignées en kinyarwanda au premier cycle et en anglais au deuxième cycle.

$\mathrm{Au}$ secondaire toujours, la configuration actuelle présente une combinaison de trois matières par section.

Le domaine des sciences humaines comporte cinq sections :

- histoire-économie-anglais ;

- anglais-économie-géographie ;

- histoire-économie-géographie ;

- anglais-français-kinyarwanda ;

- anglais-kiswahili-kinyarwanda. laissant une marge de liberté importante à des acteurs de plus en plus diversifiés sur les
moyens pour le faire. Trois types de pratiques attestent de cette tendance. concours national d'attribution des places, des diplômes et des bourses avait été longtemps la seule incitation à respecter les programmes officiels, on observe actuellement une montée en puissance d'évaluations externes se matérialisant notamment sous la forme d'un classement des écoles en fonction de la performance des élèves. a trait à la gestion des institutions publiques au moyen des contrats de performance consistant à définir, pour chaque domaine d'intervention, les objectifs opérationnels à atteindre, les indicateurs de réalisation, les échéanciers, les personnes responsables et les 
moyens d'évaluation qui seront utilisés pour attester de l'atteinte ou non des résultats attendus.

Le troisième type de pratique se rapporte au renforcement des mesures d'accréditation de nouveaux établissements. Désormais, plus aucun nouvel établissement ne peut ouvrir ses portes avant d'avoir reçu l'accord du haut conseil de l'enseignement supérieur.

En guide de conclusion, le système éducatif rwandais est passé d'une longue période de léthargie à une " anarchie de bonnes initiatives " portées par des aspirations citoyennes légitimes. La complexification du système qui en a résulté a nécessité la mise en place de mesures de régulations institutionnelles soutenues, pour maintenir le système.

\section{BIBLIOGRAPHIE}

ERNY P. (1974) : «L'enseignement au Rwanda », Tiers-Monde, tome 15, n 59-60, p. 707-722.

Mineduc (2011): Education statistics, Kigali.

National Institute of statistics of Rwanda (2011): Statistical yearbook, édition 2011, Kigali.

RUGENGANDE J. (2008) : « Développement et diversification de l'enseignement privé au Rwanda », Éducation-Formation, e-287, avril. 


\section{ANNEXES}

\section{Données générales sur le Rwanda}

\begin{tabular}{|l|}
\hline Superficie : $26340 \mathrm{~km}^{2}$ \\
\hline Capitale : Kigali \\
\hline Population totale : 10718379 \\
\hline Taux de natalité : 2,95 \\
\hline Densité : 407 \\
\hline $\begin{array}{l}\text { Taux de croissance annuelle moyen } 2010-11: \\
7,1 \%\end{array}$ \\
\hline Revenu annuel moyen par habitant : $540 \$$ \\
\hline $\begin{array}{l}\text { Langues officielles : } \\
\text { kinyarwanda, anglais, français }\end{array}$ \\
\hline
\end{tabular}

Source : National Institute of statistics of Rwanda (2011).

\section{NOTES}

1. Quelque 800000 personnes ont été massacrées lors du génocide de 1994 au Rwanda. Voir: www.un.org/french/events/rwanda/fiche.html (NdlR).

\section{INDEX}

Mots-clés : histoire, histoire de l'éducation, politique éducative, réforme de l'enseignement, système scolaire Index géographique : Rwanda

Keywords : history, educational history, educational policy, educational reform, school system Palabras claves : historia, historia de la educación, política educacional, reforma de la educación, sistema escolar 


\section{AUTEUR}

\section{MASENGESHO KAMUZINZI}

Docteur en sciences de l'éducation de l'Université catholique de Louvain. Il enseigne à l'Université nationale du Rwanda depuis 1998. Il s'intéresse aux politiques d'éducation en Afrique dans un contexte de plus en plus globalisé où les modèles éducatifs légitimés par les instances internationales de financement migrent rapidement d'un pays à l'autre, un phénomène qui contribue à l'homogénéisation des systèmes éducatifs africains. 